\title{
Gelsolin-like Actin-capping Protein (CapG) Overexpression in the Cytoplasm of Human Hepatocellular Carcinoma, Associated with Cellular Invasion, Migration and Tumor Prognosis
}

\author{
TSUNG-JUNG TSAI ${ }^{1}$, WEN-YING CHAO ${ }^{2}$, CHIEN-CHIN CHEN ${ }^{3,4}$, \\ YI-JU CHEN ${ }^{3}$, CHING-YEN LIN $^{5}$ and YING-RAY LEE ${ }^{2,5}$ \\ ${ }^{1}$ Department of Gastroenterology, Ditmanson Medical Foundation Chiayi Christian Hospital, Chiayi, Taiwan, R.O.C.; \\ ${ }^{2}$ Department of Nursing, Min-Hwei College of Health Care Management, Tainan, Taiwan, R.O.C.; \\ ${ }^{3}$ Department of Pathology, Ditmanson Medical Foundation Chiayi Christian Hospital, Chiayi, Taiwan, R.O.C.; \\ ${ }^{4}$ Department of Cosmetic Science, Chia-Nan University of Pharmacy and Science, Tainan, Taiwan, R.O.C.; \\ ${ }^{5}$ Department of Medical Research, Ditmanson Medical Foundation Chiayi Christian Hospital, Chiayi, Taiwan, R.O.C.
}

\begin{abstract}
Background/Aim: Human hepatocellular carcinoma (HCC) is one of the most common cancers worldwide. Patients with metastatic HCC ( $m$ HCC) show poor prognosis and high mortality. In previous reports, gelsolin-like actin-capping protein (CapG) has been demonstrated to regulate cancer invasion and metastasis in various human cancers. In this study, the expression of CapG was verified in normal and/or HCCs' specimens and HCC cell lines. Moreover, the bio-activity of CapG was also investigated. Materials and Methods: The expression of CapG was examined in HCC's tissue-array by immunohistochemical (IHC) staining. The $m R N A$ and protein of CapG in three HCC cell lines were determined using realtime RT-PCR and western blot. Moreover, a trans-well migration model and a matrigel-trans-well invasion assay were used to address the bio-activity of CapG in HCC cell lines. Results: CapG was detected in the cytoplasm of normal liver tissue and HCC specimens. Importantly, CapG expression was elevated in the HCC specimens compared to normal cases and was significantly overexpressed in $\mathrm{mHCC}$ cases compared to normal cases. Moreover, patients with
\end{abstract}

Correspondence to: Ying-Ray Lee, Ph.D., Department of Medical Research, Ditmanson Medical Foundation Chia-Yi Christian Hospital, 539 Zhongxiao Rd., Chiayi City 600, Taiwan, ROC. Tel: +886-5-2765041 Ext. 5560, Fax: +886 52765041 Ext.5555, e-mail: yingray.lee@gmail.com and Ching-Yen Lin, Ph.D., Department of Medical Research, Ditmanson Medical Foundation Chia-Yi Christian Hospital, 539 Zhongxiao Rd., Chiayi City 600, Taiwan, ROC. Tel: +886 52765041 Ext. 5555, Fax: +886 52765041 Ext.5555, e-mail: jouyuan22@gmail.com

Key Words: CapG, human hepatocellular carcinoma, invasion, migration. highly expressed CapG showed greater mortality in HCC cases. In addition, the RNA and protein levels of CapG among three HCC cell lines showed a positive association with cellular migration and invasive ability. CapG knockdown with shRNA in HCC cells also verified this finding. Conclusion: In the present study, it is demonstrated that CapG is expressed in the cytoplasm and could be used as a prognostic or diagnostic biomarker for $\mathrm{mHCC}$ in clinical specimens. Moreover, CapG might contribute to tumor motility and cancer-associated mortality.

HCC is one of the most common cancers in males and females, and is globally the third leading cause of cancerassociated mortality (1). Persistent chronic infection with hepatitis $\mathrm{B}$ or $\mathrm{C}$ virus is the major risk factor associated with HCC. Moreover, severe alcohol intake, non-alcoholic steatohepatitis, aflatoxin B1 exposure, haemochromatosis, obesity, diabetes, and other metabolic factors are additional risk factors that can predispose people to cause HCC (2). Although the mounting progresses in clinical treatment for $\mathrm{HCC}$ in recent years, the 5-year survival rate of $\mathrm{HCC}$ patients is still poor. There are about 1 million deaths annually caused by HCC (1). Moreover, the high rate of recurrence after surgery and uncontrolled tumor metastasis, frequent intrahepatic propagation and extrahepatic progression lead to a poor prognosis of HCC patients. Therefore, identification or improvement of the prognostic predictors and key molecular mechanisms that influence the metastasis and progression of HCC will be essential to the successful treatment (3).

CapG, a member of the gelsolin protein family, has been found in the cytoplasm and nucleus (4). It is involved in the shaping of the cellular cytoskeleton by remodeling actin filaments. CapG modulates actin length by capping its plus or 
barbed ends in a $\mathrm{Ca}^{2+}$ and PIP2-dependent manner (5). Furthermore, the biological activities of CapG include macrophage motility, membrane ruffling, phagocytosis, and vesicle rocketing (6). Previously, CapG has been identified as an oncogene in various carcinomas, including melanoma, prostate, pancreatic, breast, ovarian, gastric cancers, and lung adenocarcinoma (7-12). It is overexpressed in these cancer specimens or cell lines and has been reported to promote tumor invasion and metastasis (9). However, Watari et al. have reported that CapG expression was lost in certain cancer cell lines, including human stomach, cutaneous melanoma, smallcell lung cancer and lung adenocarcinoma cells (13). Therefore, the biological function of CapG in tumorigenesis is still unclear. In addition, CapG overexpression in the human HCC specimens has been revealed by a 2D-DIGE proteomic study (14). Patients with venous invasion showed CapG overexpression, while downregulation of CapG with siRNA reduced cellular invasion in vitro. Although this previous report demonstrated that CapG was overexpressed in the HCC total cell lysate (14), the location of CapG and the difference in expression of CapG between tumor and adjacent normal cells are still unclear. Therefore, evaluation of the expression of CapG by IHC staining in human HCC specimens is required.

\section{Materials and Methods}

Patients and tissue samples. A human liver cancer-metastasisnormal tissue-microarray (TMA) slide (CSA5) was purchased from SuperBioChips Laboratories (Seoul, Republic of Korea). A total of 45 HCC patients (including 8 patients with metastatic HCC and 37 patients with primary tumor) who were pathologically diagnosed with HCC and 9 normal cases were selected and included in this slide (The data sheet: https://www.dropbox.com/s/q9art53pxsd13sa/ CSA5.pdf?dl=0). In addition, there was one small cell neuroendocrine carcinoma case, and four cholangiocarcinoma cases in this slide, and were excluded in this study. Among HCC specimens in this slide, there were seven cases with no tumor cells. The diagnosis with tumor stage and pTNM in these cases was based on the AJCC Cancer Staging Manual (7th edition), and could be found in the data sheet. Moreover, the prognosis of these cases could also be found in the Figure 1 of this TMA. This study was approved by the Institutional Review Board (IRB) of Ditmanson Medical Foundation Chiayi Christian Hospital (IRB106018).

Cell lines and cell culture. Human hepatoma cell lines, including Huh7, Hep3B and HepG2, were obtained from American Type Culture Collection (ATCC, Manassas, VA, USA). All of the cells were cultured in Dulbecco's Modified Eagle's Medium (DMEM; Gibco BRL, Grand Island, NY, USA), and were supplemented with $10 \%$ fetal bovine serum (FBS; Trace Biosciences, Sydney, Australia), penicillin (200 U/ml; Biowest, Nuaillé, France) and streptomycin $\left(100 \mu \mathrm{g} / \mathrm{ml}\right.$; Bioweat). Cells were maintained at $37^{\circ} \mathrm{C}$ with $5 \% \mathrm{CO}_{2}$.

Immunohistochemical staining. The tissue specimens in the TMA were de-paraffinized, boiled in citric acid, and treated with hydrogen peroxide before incubation overnight with anti-CapG primary antibody (GeneTex Inc., Hsinchu, Taiwan, ROC) at $4^{\circ} \mathrm{C}$. After washing, the TMA was incubated with polymer-horseradish peroxidase-conjugated secondary antibody at room temperature (RT) for $1 \mathrm{~h}$. Specimens were developed with diaminobenzidine (DAB) and counterstained with hematoxylin (Dako, Glostrup, Denmark), and images were determined with Olympus microscopy. The intensity of staining was graded from 0 (undetectable), 1 (weak staining), 3 (medium staining) to 5 (intense staining) by Dr. ChienChin Chen (a pathologist), while the proportion of positively stained tumor cells within a tissue was scored from $0 \%$ to $100 \%$ of tumor cells identified. The staining score was expressed as the product of intensity of staining and proportion of tumor positivity.

Real-time quantitative RT-PCR. The expression of mRNA of CapG in the three HCC cell lines was analyzed by real-time PCR. The total RNAs from Huh7, Hep3B and HepG2 cells were extracted by RNA isolation kit (GE Healthcare, Munich, Germany). The cDNAs were prepared by using Transcriptor First Strand cDNA Synthesis Kit (Roche, Mannheim, Germany), and were carried out on the CFX96 Real-Time System (Bio-Rad Laboratories Inc., Hercules, CA, USA). The conditions of real-time PCR were as follows: one cycle at $50^{\circ} \mathrm{C}$ for $2 \mathrm{~min}$, and $95^{\circ} \mathrm{C}$ for $10 \mathrm{~min}$, followed by 40 cycles of denaturation at $95^{\circ} \mathrm{C}$ for $15 \mathrm{sec}$ and annealing extension at $55^{\circ} \mathrm{C}$ for $1 \mathrm{~min}$.

The primers used included:

CapG-F: CGAACACTCAGGTGGAGATT

CapG-R: TCCAGTCCTTGAAAAATTGC

GAPDH-F: TGCACCACCAACTGCTTAGC

GAPDH-R: GGCATGGACTGTGGTCATGAG

Western blotting. Total cell lysates from HCC cell lines were extracted with RIPA buffer [containing PMSF, EGTA, aprotinin, leupeptin, Na3VO4 (Sigma, St. Louis, MO, USA), and EDTA (Merck, Darmstadt, Germany)]. Proteins were examined with 10\% sodium dodecyl sulfate polyacrylamide gel electrophoresis (SDSPAGE) and transferred to PVDF membrane (Bio-Rad) and incubated overnight with primary antibody (CapG antibody; GeneTex) at $4^{\circ} \mathrm{C}$ followed by secondary antibody staining at RT for $1 \mathrm{~h}$. Finally, the data were examined using the Biospectrum Imaging system (UVP, Upland, CA, USA). The expression levels of the proteins in the cells were quantified using Image-J software.

Migration assay and matrigel invasion assay. To evaluate the impact of $\mathrm{CapG}$ expression on tumor cell migration and invasion, a trans-well migration model and a trans-well matrigel invasion assay model were established. The transwell migration and matrigel invasion assays were carried out to evaluate the in vitro migration or invasion ability among cell lines with differential expression of CapG protein. $1 \times 10^{5}$ cells/well were seeded and cultured in the transwell upper chamber [in the matrigel invasion assay model, the upper chamber was coated with matrigel $(0.8 \mathrm{mg} / \mathrm{ml}$; Corning, Bedford, MA, USA)] for $24 \mathrm{~h}$, and $10 \%$ FBS was then used as a chemoattractant in the bottom chamber for $24 \mathrm{~h}$. Cells migrated in the bottom chamber were stained with $2 \%$ crystal violet solution (Sigma, MO, USA). Moreover, control shRNA (CCGGACACT CGAGCACTTTTTG) and CapG shRNA (CCGGCCGAACAC TCAGGTGGAGATTCTCGAGAATCTCCACCTGAGTGTTCGGT TTTT) were transfected into Hep3B cells, and the cellular migration was determined under trans-well migration assay.

Statistical analysis. All data were analyzed using Graph Pad Prism for Windows, version 6 (Graph Pad Software Inc., San 


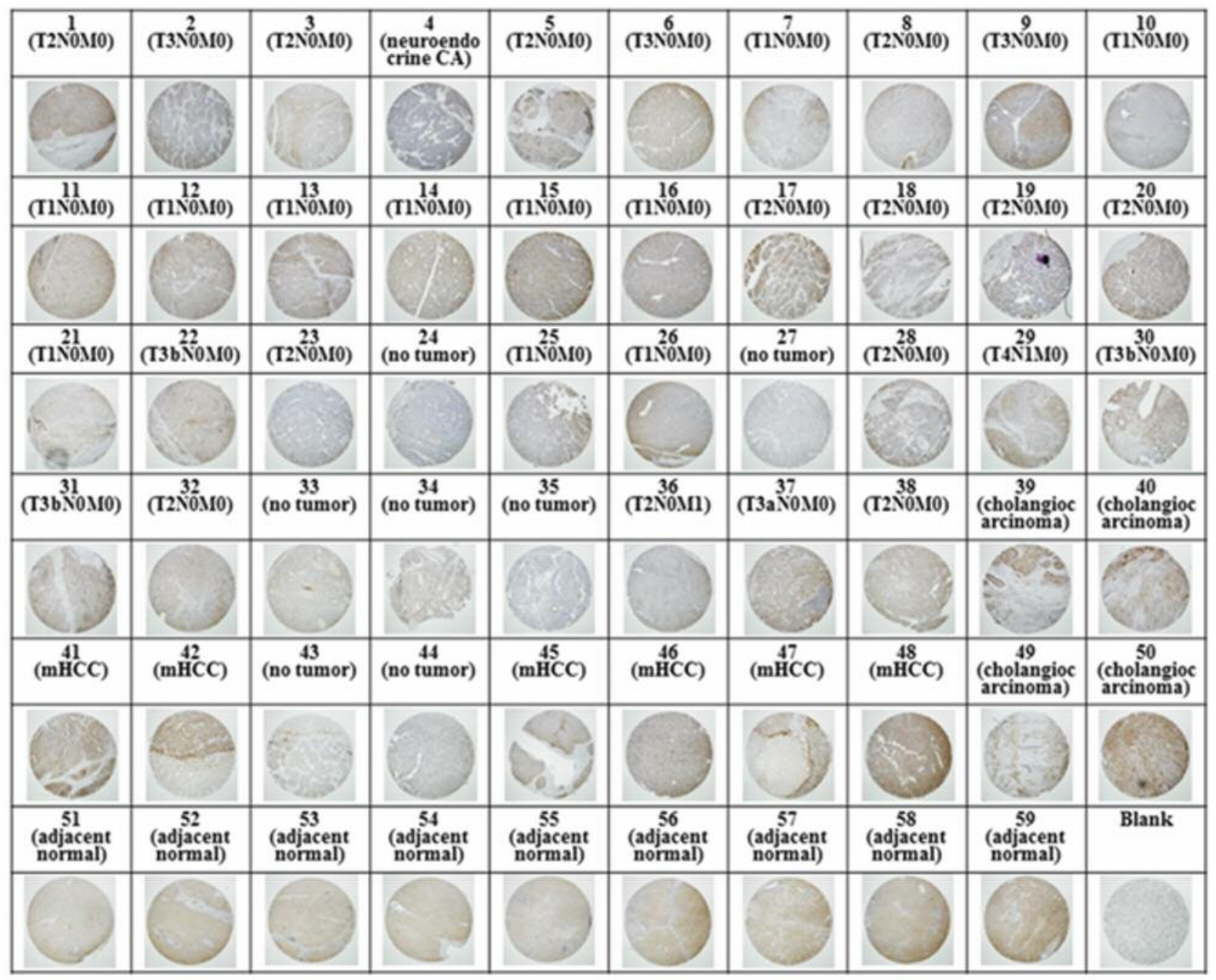

Figure 1. The expression of CapG in human hepatocellular carcinoma and normal specimens in tissue microarray. CapG in the clinical specimens was examined by immunohistochemistry, and the photographs were determined under microscopy $(\times 40)$. There were no tumor specimens in samples No. 24, No. 27, No. 33, No. 34, No. 35, No.43, and No.44. In addition, tumor specimens No. 4, No. 39, No. 40, No. 49, and No. 50 were not HCC cases. A blank is shown in No. 60.

Diego, CA, USA). The data are presented as the mean \pm SD. $p$ Values were calculated by Microsoft Excel and SPSS, version 21.0 (IBM SPSS Statistics, USA) through nonparametric tests. $p$ Values less than 0.05 were considered and reported as "statistically significant".

\section{Results}

CapG overexpression in metastatic human hepatocellular carcinoma. The clinical characteristics and the demographic data of the patients in this study are displayed in Table I. CapG expression index (EI) in the specimens was divided into high expression index $(\mathrm{EI} \geq 1.5)$ and low expression index $(\mathrm{EI}<1.5)$. The expression index of CapG in groups including sex, age, tumor size and invasive, lymph node status, tumor stage, and tumor differentiation showed no statistically significant difference (Table I). The clinical specimens in this study included 9 normal cases, $32 \mathrm{HCC}$ cases, and 6 metastatic HCC (mHCC) cases, and all of them were stained with CapG. The intensity and number of positively stained tumor cells were determined by Dr. ChienChin Chen using microscopy. CapG expression in tumor tissues as well as in normal tissues was scored according to the intensity of signal (non-expression: 0, weak-expression: 1, medium-expression: 3 , and intense-expression: 5), and the proportion of tumor positivity (0-100\%). CapG overexpression was found in the HCC specimens compared with normal cases (Figure 2A). Moreover, metastatic HCC cases showed significant overexpression of CapG compared 
Table I. Distribution of characteristics of patients and the expression index of CapG.

\begin{tabular}{|c|c|c|c|c|}
\hline \multirow[b]{2}{*}{ Clinical classification } & \multicolumn{3}{|c|}{ CapG immunostaining intensity } & \multirow[b]{2}{*}{$p$-Value } \\
\hline & Total number & Low (number) & High (number) & \\
\hline Gender & & & & $p=0.4263$ (male $v s$. female) \\
\hline Male & 36 & 18 & 18 & \\
\hline Female & 2 & 2 & 0 & \\
\hline Age (years) & & & & $p=0.1513(\geq 55$ vs. $<55)$ \\
\hline$\geq 55$ & 22 & 13 & 9 & \\
\hline$<55$ & 16 & 7 & 9 & \\
\hline T-primary tumor & & & & $p=0.325(\mathrm{~T} 1+\mathrm{T} 2$ vs. T3+T4) \\
\hline $\mathrm{T} 1+\mathrm{T} 2$ & 25 & 15 & 10 & \\
\hline $\mathrm{T} 3+\mathrm{T} 4$ & 9 & 3 & 6 & \\
\hline Lymph node status & & & & $p=0.414$ (negative $v s$. positive) \\
\hline Negative & 32 & 18 & 14 & \\
\hline Positive & 2 & 0 & 2 & \\
\hline Stage & & & & $p=0.354(\mathrm{I}+\mathrm{II} v s . \mathrm{III}+\mathrm{IV})$ \\
\hline $\mathrm{I}+\mathrm{II}$ & 23 & 14 & 9 & \\
\hline III+IV & 15 & 6 & 9 & \\
\hline Differentiated & & & & $p=0.262$ (pairwise comparison randomly) \\
\hline Well differentiated & 3 & 3 & 0 & \\
\hline Moderately differentiated & 20 & 10 & 10 & \\
\hline Poorly differentiated & 2 & 1 & 1 & \\
\hline
\end{tabular}

with normal cases (Figure 2A). This finding was consistent with the previous 2D-DIGE proteomic study (14). However, our data showed no significant difference in the expression of CapG between metastatic HCC and primary HCC's specimens (Figure 2A). Moreover, in the dead cases, patients with CapG overexpression showed relatively lower overall survival (Figure 2B). It can be suggested that patients with CapG overexpression have a poorer prognosis. Additionally, CapG overexpression appeared only in the cytoplasm in HCC patients' specimen (Figure 2C). In conclusion, our data suggested that CapG might be a prognostic marker for metastasis but could not be used as a diagnostic tumor marker for HCC patients.

$m R N A$ and protein expression of CapG in the human hepatocellular carcinoma cell lines. To further verify the expression and the biological effect of CapG in vitro, three HCC cell lines were examined. Huh-7, Hep3B, and HepG2 were used to investigate the mRNA and total protein levels of CapG by real-time RT-PCR and western blotting, respectively. The expression levels of CapG mRNA in these cells were Hep3B > Huh-7 > HepG2 (Figure 3A). Moreover, the different protein expression of CapG in these cell lines was consistent with the findings in mRNA expression (Figure 3B). Therefore, a differential expression of mRNA and protein of CapG in Huh-7, Hep3B and HepG2 cells was found, and the biological effects of CapG on the HCC metastasis were further explored.
Differential migration and invasion ability in the human hepatocellular carcinoma cell lines. To address whether the expression of CapG in HCC cells could modulate tumor cell migration or invasion, a transwell migration and invasion assay was applied. These three cells were cultured in the upper chamber without or with matrigel, and FBS was used as the chemoattractant in the bottom chamber for $24 \mathrm{~h}$. Hep3B had a greater migratory activity than Huh-7 and HepG2 and Huh-7 better than HepG2 (Figure 4A). Moreover, the invasion ability of these cells also revealed consistent results with cellular migration finding (Figure 4B). To verify the role of $\mathrm{CapG}$ on tumor migration, CapG shRNA was transfected into Hep3B cells (a CapG highly expressed cell line). Figure 5 shows a significant reduction in cell migration in CapG knockdown cells. Therefore, high expression of CapG in the in vitro model promotes tumor migration and invasion in HCC cells.

\section{Discussion}

CapG has been demonstrated to be expressed in the cellular cytoplasm and nucleus (4). Moreover, it has been recognized to be an oncogene in multiple carcinomas (7-12), and involved in the remodeling of actin filaments and cellular cytoskeleton. It is overexpressed in various human cancers, and has been reported to promote tumor invasion and metastasis $(9,10,15,16)$. In the previous study, Kimure et al. showed that CapG was overexpressed in the total cell 


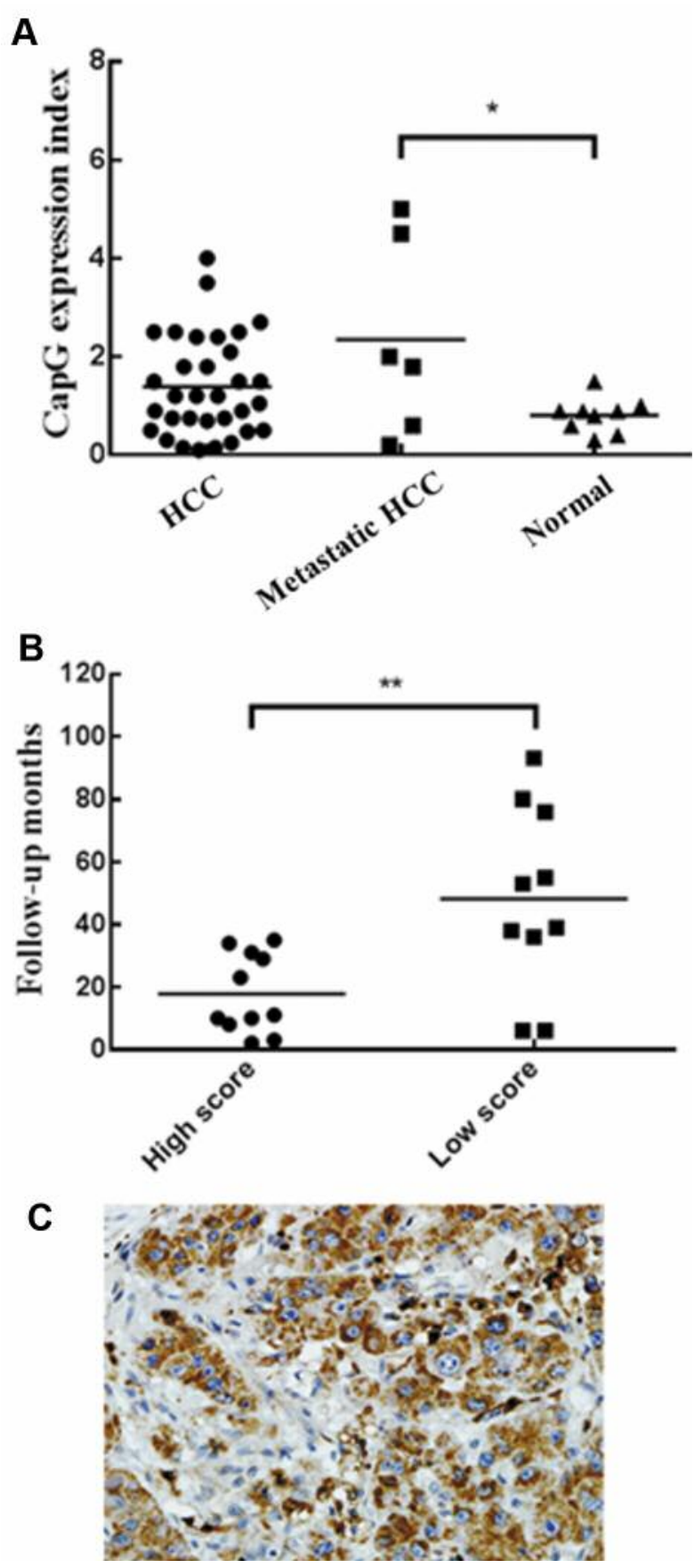

Figure 2. Expression of CapG in human hepatocellular carcinoma and normal specimens in tissue microarray. The expression of CapG was determined by immunohistochemistry in a tissue microarray, and the photographs were determined under microscopy. (A) The expression index of CapG in the HCC tissues was quantified by a pathologist and the scores of specimens were also organized depending on the pathologic diagnosis with normal, HCC, and metastatic HCC. (B) The follow-up survival month of the HCC patients, including HCC and metastatic HCC cases, were determined and grouping with the expression index of CapG (high: expression index $\geq 1.5$; low: expression index $<1.5$ ). (C) CapG expression location in the HCC tissues as shown in (A) was determined by microscopy $(\times 400)$. ${ }^{*} p<0.05, * * p<0.01, * * * p<0.001$.
A

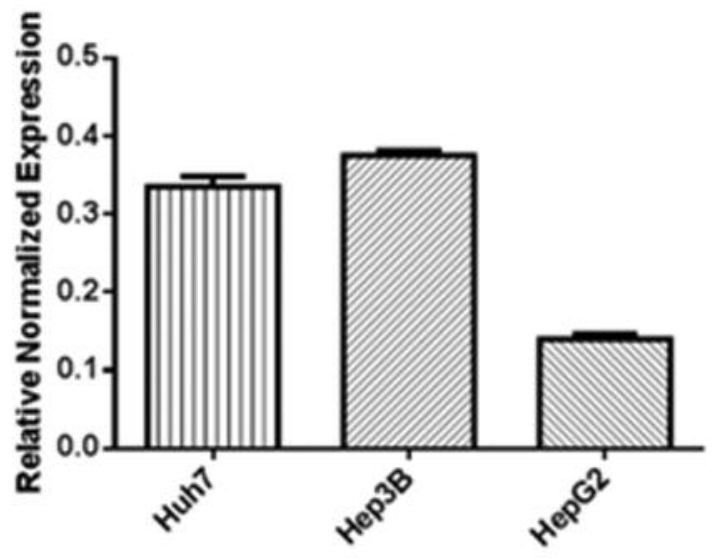

B

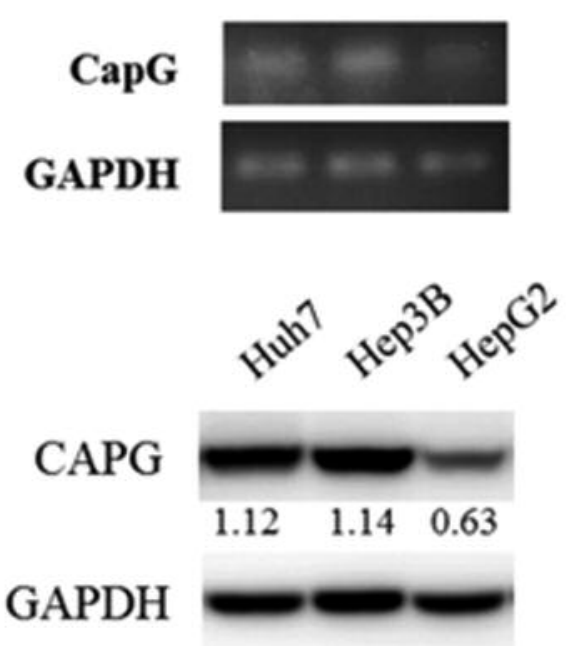

Figure 3. The mRNA and protein levels of CapG in three HCC cell lines. The expression of (A) $m R N A$ and $(B)$ protein of $C a p G$ was examined in three human HCC cell lines including Huh-7, HepG2, and Hep3B. GAPDH was used as a loading control.

lysate in the HCC tumor tissues, and was correlated with tumor invasion (14). In the present study, the expression levels and the location of CapG in the clinical specimens of human HCC were investigated by immunochemistry. CapG overexpression in the HCC was demonstrated to affect the prognosis of HCC patients (Figure 2). Furthermore, CapG overexpression might associate with the progression rather than carcinogenesis in HCC patients (Figure 2). The findings in our IHC staining data of HCC specimens were consistent with the results of the previous 2D-DIGE proteomic study 

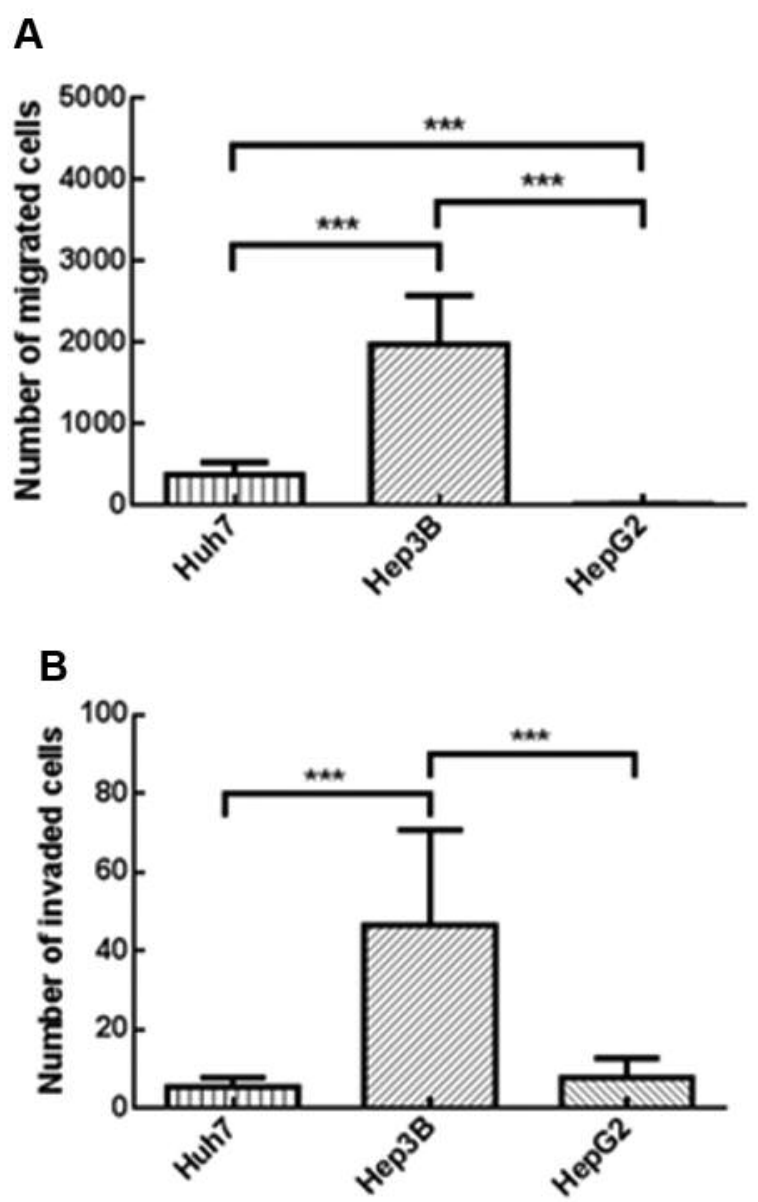

Figure 4. The behavior of cellular migration and invasion was evaluated in HCC cell lines. (A) The cellular migration activity and (B) invasion ability in Huh-7, HepG2 and Hep3B cells was determined under transwell migration assay and matrigel trans-well invasion assay after $24 \mathrm{~h}$ chemoattractant with FBS. Cells in the bottom chamber were stained with $2 \%$ crystal violet solution and the numbers were counted. $* p<0.05$, $* * p<0.01, * * * p<0.001$

(14). Importantly, our study showed that the location of CapG protein expression in the clinical HCC specimens was in the cytoplasm, while the nucleus was not remarkably involved (Figure 2C).

CapG immunostaining in the cytoplasm and/or nuclear has been reported in various human carcinomas, and had been shown to promote cancer metastasis and local invasion (17). Although the nuclear localization signal (NLS) is absent in CapG, it has been demonstrated to be regulated by calcium and phosphatidylinositol-4,5-bisphosphate (PIP2) $(18,19)$. Moreover, energy and importin- $\beta$ were demonstrated to be involved in the nuclear import of CapG (17). However, the mechanisms involved in the CapG nuclear import are still unclear. It was reported that overexpression of nuclear CapG promoted tumor invasion (17). However, overexpression of CapG in the pancreatic cancer cells was observed in the cytoplasm and the nuclear membrane, but was not detected in the nucleus (11). Moreover, the cell cycle as well as cellular proliferation had no influence on CapG overexpression in the pancreatic cancer cells, and it only affected cellular motility (11). In the present study, overexpression and non-nuclear import of CapG were found in the human $\mathrm{HCC}$ and $\mathrm{mHCC}$ specimens (Figures 1 and 2C) and CapG was associated with the prognosis and overall survival of HCC patients (Figure 2A and B).

To further examine the role of CapG in human HCC cells, the expression of mRNA and protein of CapG in Huh-7, HepG2 and Hep3B cells were investigated. The cellular migration and invasion behavior were also observed. Hep3B cells exhibited high expression of CapG and had a higher ability to migrate and invade (Figures 3 and 4). Among them, HepG2 revealed the lowest CapG expression (both in mRNA and protein level) and expressed the lowest motility and invasion activity (Figures 3 and 4). Hep3B cells expressed the highest levels of CapG protein, and knockdown of CapG with shRNA in Hep3B cells reduced cellular migration (Figure 5). The results suggested that higher CapG expression might contribute to more aggressive tumor migration and invasion. Moreover, our data were also consistent with the published reports in various human cancers $(9-11,14,20,21)$.

It was reported that enhancing or silencing CapG in the pancreatic cancer cells and HCC cells could regulate cellular invasion, but not affect cell proliferation $(11,14)$. Moreover, one previous study demonstrated that increasing importin- $\beta$ dependent nuclear import of CapG could promote tumor invasion (17). Although, CapG overexpression was localized in the cytoplasm in HCC specimens (Figure 2C) and cell lines (data not shown), our in vitro data demonstrated that cells with higher levels of CapG aggravated cellular motility and invasiveness (Figure 4). Here, we still can not conclude no other proteins that coordinate with CapG to up-regulate cellular proliferation and aggressiveness in human HCCs.

In conclusion the present study, demonstrated that CapG overexpression in the cytoplasm of human HCC is associated with poorer cancer prognosis and patients' survival. Moreover, tumor motility, as well as invasiveness is positively related to the expression levels of CapG in human HCCs.

\section{Conflicts of Interest}

The Authors declare that they have no competing interests.

\section{Acknowledgements}

This work was supported by the Grants from Chiayi Christian Hospital (Grant R106-02), and Min-Hwei College of Health Care Management. 
A

$$
\begin{aligned}
& \text { Control CapG } \\
& \text { shRNA shRNA }
\end{aligned}
$$

\section{CapG}

GAPDH

\section{B}

\section{Control shRNA}

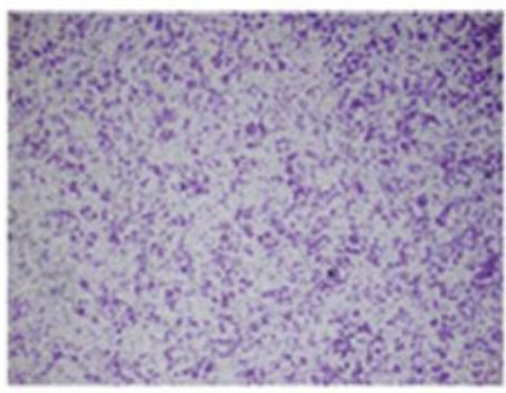

\section{CapG shRNA}

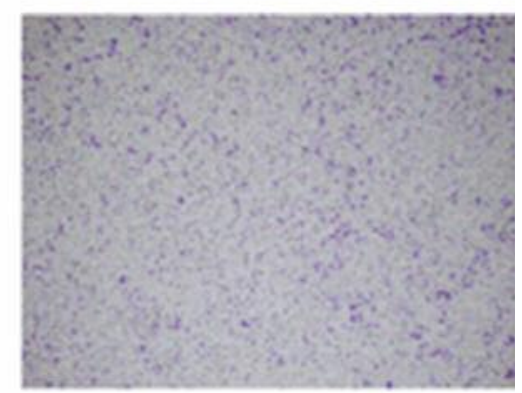

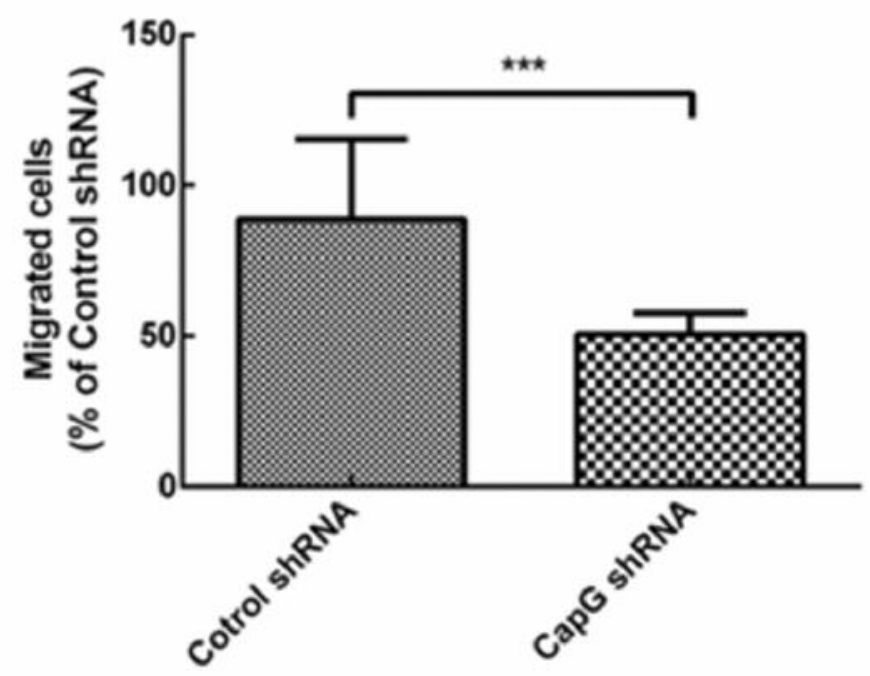

Figure 5. Knockdown of CapG reduced cellular migration of Hep3B cells. (A) Hep3B cell were transfected with a control shRNA or a CapG-shRNA, and the CapG expression was determined by western blot. (B) The cellular migration was also examined by a trans-well migration assay. GAPDH was used as a loading control. ${ }^{*} p<0.05, * * p<0.01, * * * p<0.001$.

\section{References}

1 Siegel RL, Miller KD and Jemal A: Cancer statistics, 2015. CA Cancer J Clin 65(1): 5-29, 2015.

2 Thorgeirsson SS and Grisham JW: Molecular pathogenesis of human hepatocellular carcinoma. Nature Genet 31(4): 339-346, 2002.

3 Maluccio M and Covey A: Recent progress in understanding, diagnosing, and treating hepatocellular carcinoma. CA Cancer J Clin 62(6): 394-399, 2012.

4 Onoda K, Yu FX and Yin HL: gCap39 is a nuclear and cytoplasmic protein. Cell Motil Cytoskeleton 26(3): 227-238, 1993.

5 Sun HQ, Kwiatkowska K, Wooten DC and Yin HL: Effects of CapG overexpression on agonist-induced motility and second messenger generation. J Cell Biol 129(1): 147-156, 1995.

6 Witke W, Li W, Kwiatkowski DJ and Southwick FS: Comparisons of CapG and gelsolin-null macrophages: Demonstration of a unique role for CapG in receptor-mediated ruffling, phagocytosis, and vesicle rocketing. J Cell Biol 154(4): 775-784, 2001.
7 Li T, Hong X, Zhao J, Teng Y, Zheng J, Chen H, Chen $\mathrm{H}$ and Li $\mathrm{H}$ : Gelsolin-like actin-capping protein is associated with patient prognosis, cellular apoptosis and proliferation in prostate cancer. Biomark Med 10(12): 1251-1260, 2016.

8 Westbrook JA, Cairns DA, Peng J, Speirs V, Hanby AM, Holen I, Wood SL, Ottewell PD, Marshall H, Banks RE, Selby PJ, Coleman RE and Brown JE: CAPG and GIPC1: Breast cancer biomarkers for bone metastasis development and treatment. J Nat Cancer Inst 108(4): 2016. doi: 10.1093/jnci/djv360

9 Glaser J, Neumann MH, Mei Q, Betz B, Seier N, Beyer I, Fehm T, Neubauer H, Niederacher D and Fleisch MC: Macrophage capping protein $\mathrm{CapG}$ is a putative oncogene involved in migration and invasiveness in ovarian carcinoma. BioMed Res Int 2014: 379847, 2014.

10 Ichikawa H, Kanda T, Kosugi S, Kawachi Y, Sasaki H, Wakai T and Kondo T: Laser microdissection and two-dimensional difference gel electrophoresis reveal the role of a novel macrophage-capping protein in lymph node metastasis in gastric cancer. J Proteom Res 12(8): 3780-3791, 2013. 
11 Tonack S, Patel S, Jalali M, Nedjadi T, Jenkins RE, Goldring C, Neoptolemos $\mathrm{J}$ and Costello E: Tetracycline-inducible protein expression in pancreatic cancer cells: effects of CapG overexpression. World journal of gastroenterology 17(15): 19471960, 2011.

12 Shao F, Zhang R, Don L and Ying K: Overexpression of gelsolin-like actin-capping protein is associated with progression of lung adenocarcinoma. The Tohoku J Exp Med 225(2): 95-101, 2011

13 Watari A, Takaki K, Higashiyama S, Li Y, Satomi Y, Takao T, Tanemura A, Yamaguchi Y, Katayama I, Shimakage M, Miyashiro I, Takami K, Kodama K and Yutsudo M: Suppression of tumorigenicity, but not anchorage independence, of human cancer cells by new candidate tumor suppressor gene CapG. Oncogene 25(56): 7373-7380, 2006.

14 Kimura K, Ojima H, Kubota D, Sakumoto M, Nakamura Y, Tomonaga T, Kosuge T and Kondo T: Proteomic identification of the macrophage-capping protein as a protein contributing to the malignant features of hepatocellular carcinoma. J Proteomics 78: 362-373, 2013.

15 Zhu WY, Hunag YY, Liu XG, He JY, Chen DD, Zeng F, Zhou JH and Zhang YK: Prognostic evaluation of CapG, gelsolin, Pgp, GSTP1, and Topo-II proteins in non-small cell lung cancer. Anat Rec 295(2): 208-214, 2012.

16 Kang S, Kim MJ, An H, Kim BG, Choi YP, Kang KS, Gao MQ, Park H, Na HJ, Kim HK, Yun HR, Kim DS and Cho NH: Proteomic molecular portrait of interface zone in breast cancer. J Proteome Res 9(11): 5638-5645, 2010.

17 De Corte V, Van Impe K, Bruyneel E, Boucherie C, Mareel M, Vandekerckhove $\mathrm{J}$ and Gettemans $\mathrm{J}$ : Increased importin-betadependent nuclear import of the actin modulating protein CapG promotes cell invasion. J Cell Sci 117(Pt 22): 5283-5292, 2004.
18 Johnston PA, Yu FX, Reynolds GA, Yin HL, Moomaw CR, Slaughter CA and Sudhof TC: Purification and expression of gCap39. An intracellular and secreted $\mathrm{Ca} 2(+)$-dependent actinbinding protein enriched in mononuclear phagocytes. J Biol Chem 265(29): 17946-17952, 1990.

19 Yu FX, Johnston PA, Sudhof TC and Yin HL: gCap39, a calcium ion- and polyphosphoinositide-regulated actin capping protein. Science 250(4986): 1413-1415, 1990.

20 Van Impe K, Bethuyne J, Cool S, Impens F, Ruano-Gallego D, De Wever O, Vanloo B, Van Troys M, Lambein K, Boucherie C, Martens E, Zwaenepoel O, Hassanzadeh-Ghassabeh G, Vandekerckhove J, Gevaert K, Fernandez LA, Sanders NN and Gettemans J: A nanobody targeting the F-actin capping protein CapG restrains breast cancer metastasis. Breast Cancer Res 15(6): R116, 2013.

21 Morofuji N, Ojima H, Onaya H, Okusaka T, Shimada K, Sakamoto Y, Esaki M, Nara S, Kosuge T, Asahina D, Ushigome M, Hiraoka N, Nagino $M$ and Kondo T: Macrophage-capping protein as a tissue biomarker for prediction of response to gemcitabine treatment and prognosis in cholangiocarcinoma. $\mathbf{J}$ Proteom 75(5): 1577-1589, 2012. 